\title{
LETTER
}

\section{Findings of lung ultrasonography of novel corona virus pneumonia during the 2019-2020 epidemic}

\author{
Qian-Yi Peng ${ }^{1}$, Xiao-Ting Wang ${ }^{2^{*}}$, Li-Na Zhang ${ }^{1 *}$ and Chinese Critical Care Ultrasound Study Group (CCUSG)
}

(C) 2020 Springer-Verlag GmbH Germany, part of Springer Nature

Dear Editor,

Up to 24 February 2020, there have been 77,269 officially reported confirmed cases of 2019 novel corona virus (SARS-CoV-2) infection in China. As lung abnormalities may develop before clinical manifestations and nucleic acid detection, experts have recommended early chest computerized tomography $(\mathrm{CT})$ for screening suspected patients [1]. The high contagiousness of SARS$\mathrm{CoV}-2$ and the risk of transporting unstable patients with hypoxemia and hemodynamic failure make chest CT a limited option for the patient with suspected or established COVID-19. Lung ultrasonography gives the results that are similar to chest $\mathrm{CT}$ and superior to standard chest radiography for evaluation of pneumonia and/ or adult respiratory distress syndrome (ARDS) with the added advantage of ease of use at point of care, repeatability, absence of radiation exposure, and low cost [2].

In this report, we summarize our early experience with lung ultrasonography for evaluation of SARS-CoV-2 infection in China with the intent of alerting frontline intensivists to the utility of lung ultrasonography for management of COVID-19.

\section{Ultrasonographic features of $\mathbf{n C o V}$ pneumonia}

We performed lung ultrasonography on 20 patients with COVID-19 using a 12-zone method [3].

\footnotetext{
*Correspondence: icuting@163.com; zln7095@163.com

${ }^{1}$ Department of Critical Care Medicine, Xiangya Hospital, Central South University, No. 87 Xiangya Road, Changsha 410008, Hunan Province, China

${ }^{2}$ Department of Critical Care Medicine, Peking Union Medical College Hospital, Tsinghua University, No.1 Shuaifuyuan, Wangfujing, Dongcheng District 100730, Beijing, China

Full author information is available at the end of the article
}

Characteristic findings included the following:

1. Thickening of the pleural line with pleural line irregularity;

2. B lines in a variety of patterns including focal, multifocal, and confluent;

3. Consolidations in a variety of patterns including multifocal small, non-translobar, and translobar with occasional mobile air bronchograms;

4. Appearance of A lines during recovery phase;

5. Pleural effusions are uncommon.

The observed patterns occurred across a continuum from mild alveolar interstitial pattern, to severe bilateral interstitial pattern, to lung consolidation. Table 1 summarizes typical lung ultrasonography finds in patients with COVID-19 respiratory disease in comparison with chest CT findings. Typical lung ultrasonography images are shown in the supplementary material (Supplementary Fig. 1.)

The findings of lung ultrasonography features of SARS$\mathrm{CoV}-2$ pneumonia/ARDS are related to the stage of disease, the severity of lung injury, and comorbidities. The predominant pattern is of varying degrees of interstitial syndrome and alveolar consolidation, the degree of which is correlated with the severity of the lung injury. A recognized limitation of lung ultrasonography is that it cannot detect lesions that are deep within the lung, as aerated lung blocks transmission of ultrasonography, i.e., the abnormality must extend to the pleural surface to be visible with on ultrasonography examination. Chest $\mathrm{CT}$ is required to detect pneumonia that does not extend to the pleural surface. 
Table 1 CT and ultrasonographic features of COVID-19 pneumonia

\begin{tabular}{|c|c|}
\hline Lung CT & Lung ultrasound \\
\hline Thickened pleura & Thickened pleural line \\
\hline Ground glass shadow and effusion & B lines (multifocal, discrete, or confluent) \\
\hline Pulmonary infiltrating shadow & Confluent B lines \\
\hline Subpleural consolidation & Small (centomeric) consolidations) \\
\hline Translobar consolidation & Both non-translobar and translobar consolidation \\
\hline Pleural effusion is rare. & Pleural effusion is rare \\
\hline More than two lobes affected & Multilobar distribution of abnormalities \\
\hline $\begin{array}{l}\text { Negative or atypical in lung CT images in the super-early stage, then dif- } \\
\text { fuse scattered or ground glass shadow with the progress of the disease, } \\
\text { further lung consolidation }\end{array}$ & $\begin{array}{l}\text { Focal B lines is the main feature in the early stage and in mild infection; } \\
\text { alveolar interstitial syndrome is the main feature in the progressive stage } \\
\text { and in critically ill patients; A lines can be found in the convalescence; } \\
\text { pleural line thickening with uneven B lines can be seen in patients with } \\
\text { pulmonary fibrosis }\end{array}$ \\
\hline
\end{tabular}

Based upon our experience, we consider that lung ultrasonography has major utility for management of COVID-19 with respiratory involvement due to its safety, repeatability, absence of radiation, low cost and point of care use; chest CT may be reserved for cases where lung ultrasonography is not sufficient to answer the clinical question. We find there is utility of lung ultrasonography for rapid assessment of the severity of SARS-CoV-2 pneumonia/ARDS at presentation, to track the evolution of disease, to monitor lung recruitment maneuvers, to guide response to prone position, the management of extracorporeal membrane therapy, and for making decisions related to weaning the patient form ventilatory support.

\section{Electronic supplementary material}

The online version of this article (https://doi.org/10.1007/s00134-020-05996-6) contains supplementary material, which is available to authorized users.

\section{Author details \\ ${ }^{1}$ Department of Critical Care Medicine, Xiangya Hospital, Central South University, No. 87 Xiangya Road, Changsha 410008, Hunan Province, China. 2 Department of Critical Care Medicine, Peking Union Medical College Hospital, Tsinghua University, No.1 Shuaifuyuan, Wangfujing, Dongcheng District 100730, Beijing, China.}

\section{Funding}

Funding was provided by National Natural Science Foundation of China (Grant no. 81873956) and National Natural Science Foundation of China (Grant no. 81974285).

\section{Compliance with ethical standards}

\section{Conflicts of interest}

On behalf of all authors, the corresponding author states that there is no conflict of interest.

\section{Publisher's Note}

Springer Nature remains neutral with regard to jurisdictional claims in published maps and institutional affiliations.

Accepted: 2 March 2020

Published online: 12 March 2020

\section{References}

1. National Health Commission of the people's Republic of China. Diagnosis and treatment of novel coronavirus pneumonia (trial, the fifth version) [EB/OL]. (2020-02-05)[2020-02-06]. http://www.nhc.gov.cn/yzygj/s7653 p/202002/3b09b894ac9b4204a79db5b8912d4440.shtml

2. Mayo PH, Copetti R, Feller-Kopman D, Mathis G, Maury E, Mongodi S, Mojoli F, Volpicelli G, Zanobetti M (2019) Thoracic ultrasonography: a narrative review. Intensive Care Med 45:1200-1211. https://doi.org/10.1007/ s00134-019-05725-8

3. Soummer A, Perbet S, Brisson H, Arbelot C, Constantin JM, Lu Q, Rouby JJ, Lung Ultrasound Study Group (2012) Ultrasound assessment of lung aeration loss during a successful weaning trial predicts postextubation distress. Crit Care Med. 40(7):2064-2072. https://doi.org/10.1097/ CCM.0b013e31824e68ae 